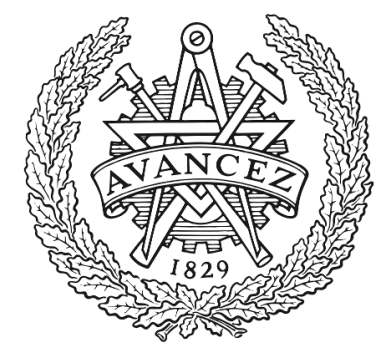

CHALMERS

UNIVERSITY OF TECHNOLOGY

\title{
Counting rational points on smooth cubic curves
}

Downloaded from: https://research.chalmers.se, 2023-04-26 12:10 UTC

Citation for the original published paper (version of record):

Tran, M. (2018). Counting rational points on smooth cubic curves. Journal of Number Theory, 189: 138-146. http://dx.doi.org/10.1016/j.jnt.2017.12.001

N.B. When citing this work, cite the original published paper. 


\title{
Counting rational points on smooth cubic curves
}

\author{
Manh Hung Tran
}

Department of Mathematical Sciences, Chalmers University of Technology, Sweden

\section{A R T I C L E I N F O}

\section{Article history:}

Received 26 October 2016

Received in revised form 6 December 2017

Accepted 7 December 2017

Available online 2 February 2018

Communicated by A. Pal

Keywords:

Cubic curves

Rational points

Counting function

Elliptic curves

Global determinant method

Descent
A B S T R A C T

We use a global version of Heath-Brown's $p$-adic determinant method developed by Salberger to give upper bounds for the number of rational points of height at most $B$ on non-singular cubic curves defined over $\mathbb{Q}$. The bounds are uniform in the sense that they only depend on the rank of the corresponding Jacobian.

(c) 2018 Elsevier Inc. All rights reserved.

\section{Introduction}

Let $F\left(X_{0}, X_{1}, X_{2}\right) \in \mathbb{Z}\left[X_{0}, X_{1}, X_{2}\right]$ be a non-singular cubic form, so that $F=0$ defines a smooth plane cubic curve $C$ in $\mathbb{P}^{2}$. We want to study the asymptotic behaviour of the counting function

$$
N(B)=\sharp\{P \in C(\mathbb{Q}): H(P) \leq B\},
$$

with respect to the naive height function $H(P):=\max \left\{\left|x_{0}\right|,\left|x_{1}\right|,\left|x_{2}\right|\right\}$ for $P=\left[x_{0}, x_{1}, x_{2}\right]$ with co-prime integer values of $x_{0}, x_{1}, x_{2}$.

E-mail address: manhh@chalmers.se. 
It is known that if the rank $r$ of the $\operatorname{Jacobian} \operatorname{Jac}(C)$ is positive, then we have

$$
N(B) \sim c_{F}(\log B)^{r / 2}
$$

as $B \rightarrow \infty$. This result was shown by Néron. Moreover, if $r=0$ then $N(B) \leq 16$ by Mazur's theorem (see Mazur [5], Theorem 8) on torsion groups of elliptic curves. But (1) is not a uniform upper bound as the constant $c_{F}$ depends on $C$. The aim of this paper is to give uniform upper bounds for $N(B)$ which only depend on the rank of $\operatorname{Jac}(C)$.

In this direction, Heath-Brown and Testa (see [4], Corollary 1.3) established the uniform bound

$$
N(B) \ll(\log B)^{3+r / 2}
$$

by using the $p$-adic determinant method developed by the first author (see [3]). In [4], they also used a result of David [1] about the successive minima of the quadratic form given by the canonical height pairing on $\operatorname{Jac}(C)$ to prove the sharper uniform bounds $N(B) \ll(\log B)^{1+r / 2}$ for all $r$ and $N(B) \ll(\log B)^{r / 2}$ if $r$ is sufficiently large.

We shall in this paper give a direct proof of the bound

$$
N(B) \ll(\log B)^{2+r / 2},
$$

based on the determinant method, which does not depend on any deep result about the canonical height pairing.

To do this, we follow the approach in [4] with descent. But we replace the $p$-adic determinant method by a global determinant method developed by Salberger [6]. The main result of this paper is the following

Theorem 1. Let $F\left(X_{0}, X_{1}, X_{2}\right) \in \mathbb{Z}\left[X_{0}, X_{1}, X_{2}\right]$ be a non-singular cubic form, so that $F=0$ defines a smooth plane cubic curve $C$. Let $r$ be the rank of $J a c(C)$. Then for any $B \geq 3$ and any positive integer $m$ we have

$$
N(B) \ll m^{r}\left(B^{\frac{2}{3 m^{2}}}+m^{2}\right) \log B
$$

uniformly in $C$, with an implied constant independent of $m$.

This bound improves upon the estimate

$$
N(B) \ll m^{r+2}\left(B^{\frac{2}{3 m^{2}}} \log B+\log ^{2} B\right)
$$

in [4] (see Theorem 1.2). Taking $m=1+[\sqrt{\log B}]$ we immediately obtain the following result. 
Corollary 2. Under the conditions above we have

$$
N(B) \ll(\log B)^{2+r / 2}
$$

uniformly in $C$.

In the appendix we include for comparison a short account of the bounds for $N(B)$ that can be deduced from David's result.

\section{The descent argument}

We shall in this section recall the argument in [4], where the study of $N(B)$ is reduced to a counting problem for a biprojective curve.

Let $\psi: C \times C \rightarrow \operatorname{Jac}(C)$ be the morphism to the Jacobian of $C$ defined by $\psi(P, Q)=$ $[P]-[Q]$. Let $m$ be a positive integer and define an equivalence relation on $C(\mathbb{Q})$ as follows: $P \sim_{m} Q$ if $\psi(P, Q) \in m(\operatorname{Jac}(C)(\mathbb{Q}))$. The number of equivalence classes is at most $16 \mathrm{~m}^{r}$ by the theorems of Mazur and Mordell-Weil. There is therefore a class $K$ such that

$$
N(B) \ll m^{r} \sharp\{P \in K: H(P) \leq B\} .
$$

If we fix a point $R$ in $K$ then for any other point $P$ in $K$, there will be a further point $Q$ in $C(\mathbb{Q})$ such that $[P]=m[Q]-(m-1)[R]$ in the divisor class group of $C$. We define the curve $X=X_{R}$ by

$$
X_{R}:=\{(P, Q) \in C \times C:[P]=m[Q]-(m-1)[R]\}
$$

in $\mathbb{P}^{2} \times \mathbb{P}^{2}$. Then $N(B) \ll m^{r} \sharp \mathcal{K}$, where

$$
\mathcal{K}:=\{(P, Q) \in X(\mathbb{Q}): H(P) \leq B\}
$$

We have thus reduced the counting problem for $C$ to a counting problem for a biprojective curve $X$ in $\mathbb{P}^{2} \times \mathbb{P}^{2}$. We shall also need the following lemma from [4] (see Lemma 2.1).

Lemma 3. Let $C$ be a smooth plane cubic curve defined by a primitive form $F$ with $\|F\| \ll B^{30}$, and $R$ be a point in $C(\mathbb{Q})$. There exists an absolute constant $A$ with the following property. Suppose that $(P, Q)$ is a point in $X_{R}(\mathbb{Q})$ and that $B \geq 3$. Then if $H(P), H(R) \leq B$ we have $H(Q) \leq B^{A}$.

\section{The global determinant method}

We shall in this section apply Salberger's global determinant method in [6] to $X$ and consider congruences between integral points on $X$ modulo all primes of good reduction for $C$ and $X$. It is a refinement of the $p$-adic determinant method used in [3] and [4]. 
We will label the points in $\mathcal{K}$ as $\left(P_{j}, Q_{j}\right)$ for $1 \leq j \leq N$, say, and fix integers $a, b \geq 1$. Let $I_{1}$ be the vector space of all bihomogeneous forms in $\left(x_{0}, x_{1}, x_{2} ; y_{0}, y_{1}, y_{2}\right)$ of bidegree $(a, b)$ with coefficients in $\mathbb{Q}$ and $I_{2}$ be the subspace of such forms which vanish on $X$. Since the monomials

$$
x_{0}^{e_{0}} x_{1}^{e_{1}} x_{2}^{e_{2}} y_{0}^{f_{0}} y_{1}^{f_{1}} y_{2}^{f_{2}}
$$

with

$$
e_{0}+e_{1}+e_{2}=a \text { and } f_{0}+f_{1}+f_{2}=b
$$

form a basis for $I_{1}$, there is a subset of monomials $\left\{F_{1}, \ldots, F_{s}\right\}$ whose corresponding cosets form a basis for $I_{1} / I_{2}$. As in [4] (see Lemma 3.1), if $\frac{1}{a}+\frac{m^{2}}{b}<3$, then $s=3\left(m^{2} a+b\right)$. Thus we shall always assume that $a \geq 1$ and $b \geq m^{2}$ to make sure that $s=3\left(m^{2} a+b\right)$. Consider the $N \times s$ matrix

$$
M=\left(\begin{array}{cccc}
F_{1}\left(P_{1}, Q_{1}\right) & F_{2}\left(P_{1}, Q_{1}\right) & \ldots & F_{s}\left(P_{1}, Q_{1}\right) \\
F_{1}\left(P_{2}, Q_{2}\right) & F_{2}\left(P_{2}, Q_{2}\right) & \ldots & F_{s}\left(P_{2}, Q_{2}\right) \\
\vdots & \vdots & \ldots & \vdots \\
F_{1}\left(P_{N}, Q_{N}\right) & F_{2}\left(P_{N}, Q_{N}\right) & \ldots & F_{s}\left(P_{N}, Q_{N}\right)
\end{array}\right) .
$$

If we can choose $a$ and $b$ such that $\operatorname{rank}(M)<s$, then there is a non-zero column vector $\underline{c}$ such that $M \underline{c}=\underline{0}$. This will produce a bihomogeneous form $G$, say, of bidegree $(a, b)$ such that $G\left(P_{j}, Q_{j}\right)=0$ for all $1 \leq j \leq N$. Hence all points in $\mathcal{K}$ will lie on the variety $Y \subset \mathbb{P}^{2} \times \mathbb{P}^{2}$ given by $G=0$, while the irreducible curve $X$ does not lie on $Y$. Thus

$$
N \leq \sharp(X \cap Y) \leq 3\left(m^{2} a+b\right)
$$

by the Bézout-type argument in [4] (see Lemma 5.1).

In order to show that $\operatorname{rank}(M)<s$, we may clearly suppose that $N \geq s$. We will show that each $s \times s$ minor $\operatorname{det}(\Delta)$ of $M$ vanishes. Without loss of generality, let $\Delta$ be the $s \times s$ matrix formed by the first $s$ rows of $M$.

$$
\Delta=\left(\begin{array}{cccc}
F_{1}\left(P_{1}, Q_{1}\right) & F_{2}\left(P_{1}, Q_{1}\right) & \ldots & F_{s}\left(P_{1}, Q_{1}\right) \\
F_{1}\left(P_{2}, Q_{2}\right) & F_{2}\left(P_{2}, Q_{2}\right) & \ldots & F_{s}\left(P_{2}, Q_{2}\right) \\
\vdots & \vdots & \ldots & \vdots \\
F_{1}\left(P_{s}, Q_{s}\right) & F_{2}\left(P_{s}, Q_{s}\right) & \ldots & F_{s}\left(P_{s}, Q_{s}\right)
\end{array}\right)
$$

The idea is now to give an upper bound for $\operatorname{det}(\Delta)$ which is smaller than a certain integral factor of $\operatorname{det}(\Delta)$. To do this, we first recall a result from [3] (see Theorem 4).

Lemma 4. For a plane cubic curve $C$ defined by a primitive integral form $F$, either $N(B) \leq 9$ or $\|F\| \ll B^{30}$. 
Thus from now on, we may and shall always suppose that $\|F\| \ll B^{30}$. It is not difficult to see that every entry in $\Delta$ has modulus at most $B^{a} B^{A b}$, where $A$ is the absolute constant in Lemma 3. Since $\Delta$ is an $s \times s$ matrix, we get that

$$
\log |\operatorname{det}(\Delta)| \leq s \log s+s \log B^{a+A b}
$$

Now we find a factor of $\operatorname{det}(\Delta)$ of the form $p^{N_{p}}$, where $p$ is a prime of good reduction for $C$. In order to do that, we divide $\Delta$ into blocks such that elements in each block have the same reduction modulo $p$.

Let $p$ be a prime number and $Q^{*}$ be a point on $C\left(\mathbb{F}_{p}\right)$. Then we define the set

$$
S\left(Q^{*}, p, \Delta\right)=\left\{\left(P_{j}, Q_{j}\right): 1 \leq j \leq s, \overline{Q_{j}}=Q^{*}\right\}
$$

where $\overline{Q_{j}}$ denotes the reduction from $C(\mathbb{Q})$ to $C\left(\mathbb{F}_{p}\right)$. Suppose $\sharp S\left(Q^{*}, p, \Delta\right)=E$. We consider any $E \times E$ sub-matrix $\Delta^{*}$ of $\Delta$ corresponding to $S\left(Q^{*}, p, \Delta\right)$ and recall a result from [4] (see Lemma 4.2). Note that our set $S\left(Q^{*}, p, \Delta\right)$ has fewer elements than the set $S\left(Q^{\prime} ; p, B\right)$ defined at the beginning of Section 3 in [4] but the proof still works.

Lemma 5. If $p$ is a prime of good reduction for $C$, then $p^{E(E-1) / 2} \operatorname{divides} \operatorname{det}\left(\Delta^{*}\right)$.

From this lemma we obtain a factor of $\operatorname{det}(\Delta)$ of the form $p^{N_{p}}$ by means of Laplace expansion. Moreover, we can do the same argument for all primes of good reduction for $C$ and then obtain a very large factor of $\operatorname{det}(\Delta)$. That is the idea of the global determinant method in [6].

Lemma 6. Let $p$ be a prime of good reduction for $C$. There exists a non-negative integer $N_{p} \geq \frac{s^{2}}{2 n_{p}}+O(s)$ such that $p^{N_{p}} \mid \operatorname{det}(\Delta)$, where $n_{p}$ is the number of $\mathbb{F}_{p}$-points on $C\left(\mathbb{F}_{p}\right)$.

Proof. Let $P$ be a point on $C\left(\mathbb{F}_{p}\right)$ and $s_{P}$ be the number of elements in $S(P, p, \Delta)$. Then by Lemma 5 , there exists an integer $N_{P}=s_{P}\left(s_{P}-1\right) / 2$ such that $p^{N_{P}} \mid \operatorname{det}\left(\Delta^{*}\right)$ for each $s_{P} \times s_{P}$ sub-matrix $\Delta^{*}$ of $\Delta$ corresponding to $S(P, p, \Delta)$.

If we apply this to all points on $C\left(\mathbb{F}_{p}\right)$ and use Laplace expansion, then we get that $p^{N_{p}} \mid \operatorname{det}(\Delta)$ for

$$
N_{p}=\sum_{P} N_{P}=\frac{1}{2} \sum_{P} s_{P}^{2}-\frac{s}{2} \geq \frac{s^{2}}{2 n_{p}}+O(s)
$$

in case $C$ has good reduction at $p$. This completes the proof of Lemma 6 .

We now give a bound for the product of primes of bad reduction for $C$. Since $\|F\| \ll$ $B^{30}$, the discriminant $D_{F}$ of $F$ will satisfy $\log \left|D_{F}\right| \ll \log B$. Thus $\log \Pi_{C} \ll \log B$, where $\Pi_{C}$ is the product of all primes of bad reduction for $C$. We have therefore the following bound. 
Lemma 7. Suppose that $\|F\| \ll B^{30}$. The product $\Pi_{C}$ of all primes of bad reduction for $C$ satisfies $\log \Pi_{C}=O(\log B)$.

We need one more lemma from [6] (see Lemma 1.10).

Lemma 8. Let $\Pi>1$ be an integer and $p$ run over all prime factors of $\Pi$. Then

$$
\sum_{p \mid \Pi} \frac{\log p}{p} \leq \log \log \Pi+2 .
$$

Proof. We may and shall assume that $\Pi$ is a square-free. Let $l$ be a positive integer such that $l \leq \Pi$ and $v_{p}(n)$ be the highest integer such that $p^{v_{p}(n)} \mid n$. We then have (see Tenenbaum [7], pp. 13-14)

$$
\begin{gathered}
l \sum_{p \mid \Pi} \frac{\log p}{p}-\sum_{p \mid \Pi} \log p \leq \sum_{p \mid \Pi} v_{p}(l !) \log p \\
\leq \sum_{p \leq \Pi} v_{p}(l !) \log p=\log l ! \leq l \log l \\
\Rightarrow \sum_{p \mid \Pi} \frac{\log p}{p} \leq \log l+\frac{1}{l} \sum_{p \mid \Pi} \log p \leq \log l+(1 / l) \log \Pi .
\end{gathered}
$$

To obtain the assertion, let $l=[\log \Pi]$ for $\Pi>2$.

\section{Proof of Theorem 1}

We now use the lemmas in Section 3 to prove that $\operatorname{det}(\Delta)$ vanishes if $s$ is large enough. Let $\Pi_{C}$ be the product of all primes $p$ of bad reduction for $C$. Then

$$
\sum_{p \mid \Pi_{C}} \frac{\log p}{p} \leq \log \log B+O(1)
$$

by Lemma 7 and Lemma 8. We apply Lemma 6 to the primes $p \leq s$ of good reduction for $C$ and write $\sum_{p \leq s}^{*}$ for a sum over these primes. We then obtain a positive factor $T$ of $\operatorname{det}(\Delta)$ which is relatively prime to $\Pi_{C}$ such that

$$
\log T \geq \frac{s^{2}}{2} \sum_{p \leq s}^{*} \frac{\log p}{n_{p}}+O(s) \sum_{p \leq s}^{*} \log p .
$$

The last term is $O\left(s^{2}\right)$ since $\sum_{p \leq s} \log p=O(s)$ (see [7], p. 31). Also,

$$
\frac{\log p}{n_{p}} \geq \frac{\log p}{p}-\frac{\left(n_{p}-p\right) \log p}{p^{2}} .
$$


Moreover, it is well-known that if $p$ is a prime of good reduction for $C$, then $n_{p}=$ $p+O(\sqrt{p})$. Thus we conclude that

$$
\frac{\log p}{n_{p}} \geq \frac{\log p}{p}+O\left(\frac{\log p}{p^{3 / 2}}\right)
$$

for all primes $p$ of good reduction for $C$. Therefore,

$$
\sum_{p \leq s}^{*} \frac{\log p}{n_{p}} \geq \sum_{p \leq s}^{*} \frac{\log p}{p}+O(1)
$$

and then

$$
\log T \geq \frac{s^{2}}{2} \sum_{p \leq s}^{*} \frac{\log p}{p}+O\left(s^{2}\right)
$$

But by (6),

$$
\sum_{p \leq s} \frac{\log p}{p}-\sum_{p \leq s}^{*} \frac{\log p}{p} \leq \log \log B+O(1)
$$

and $\sum_{p \leq s} \frac{\log p}{p}=\log s+O(1)($ see $[7]$, p. 14). Hence,

$$
\log T \geq \frac{s^{2}}{2} \log \left(\frac{s}{\log B}\right)+O\left(s^{2}\right)
$$

Thus from (5) and (7) we obtain

$$
\begin{gathered}
\log \left(\frac{|\operatorname{det}(\Delta)|}{T}\right) \leq s \log s+s \log B^{a+A b}-\frac{s^{2}}{2} \log \left(\frac{s}{\log B}\right)+O\left(s^{2}\right) \\
=\frac{s^{2}}{2}\left(\log B^{\frac{2(a+A b)}{s}}-\log \left(\frac{s}{\log B}\right)\right)+O\left(s^{2}\right) .
\end{gathered}
$$

There is therefore an absolute constant $u \geq 1$ such that

$$
\log \left(\frac{|\operatorname{det}(\Delta)|}{T}\right) \leq \frac{s^{2}}{2}\left(\log B^{\frac{2(a+A b)}{s}}-\log \left(\frac{s}{u \log B}\right)\right)
$$

If

$$
s>u B^{\frac{2(a+A b)}{s}} \log B
$$

we have in particular that $\log \left(\frac{|\operatorname{det}(\Delta)|}{T}\right)<0$ and hence $\operatorname{det}(\Delta)=0$ as $\frac{|\operatorname{det}(\Delta)|}{T} \in \mathbb{Z}_{\geq 0}$. 
Remember that $s=3\left(m^{2} a+b\right)$ if $a \geq 1$ and $b \geq m^{2}$. We now choose $b=m^{2}$ and

$$
a=1+\left[\frac{u B^{\frac{2}{3 m^{2}}} \log B}{m^{2}}+A \log B\right]
$$

Then

$$
\begin{gathered}
u B^{\frac{2(a+A b)}{s}} \log B=u B^{\frac{2\left(a+A m^{2}\right)}{3 m^{2}(a+1)}} \log B \\
<u B^{\frac{2}{3 m^{2}}} B^{\frac{2 A}{3 a}} \log B<s .
\end{gathered}
$$

Thus (8) holds and hence $\operatorname{det}(\Delta)=0$. Then $\operatorname{rank}(M)<s$ such that there is a bihomogeneous form in $\mathbb{Q}\left[x_{0}, x_{1}, x_{2}, y_{0}, y_{1}, y_{2}\right]$ which vanishes at all $\left(P_{j}, Q_{j}\right) \in X(Q), 1 \leq j \leq N$, with $H\left(P_{j}\right) \leq B$ but not everywhere on $X$. Hence (see (4))

$$
\begin{gathered}
N \leq 3\left(m^{2} a+b\right) \ll\left(B^{\frac{2}{3 m^{2}}}+m^{2}\right) \log B \\
\Rightarrow N(B) \ll m^{r}\left(B^{\frac{2}{3 m^{2}}}+m^{2}\right) \log B .
\end{gathered}
$$

This completes the proof of Theorem 1.

\section{Acknowledgment}

I wish to thank my supervisor Per Salberger for introducing me to the problem and giving me many useful suggestions.

\section{Appendix A}

In this appendix we record the following more precise version of a result in [4].

Theorem 9. Let $C$ be any smooth plane cubic curve and $r$ be the rank of $J a c(C)$. Let $m_{l}=\frac{l^{2}-4 l-4}{8 l^{2}+8 l}$ for $l \geq 1$. Then

$$
N(B) \ll \begin{cases}(\log B)^{-\left(m_{1}+\ldots+m_{r}\right)+r / 2}, & \text { if } 1 \leq r<16 \\ (\log B)^{r / 2}, & \text { if } r \geq 16\end{cases}
$$

with an absolute implied constant. In particular, $N(B) \ll(\log B)^{1+r / 2}$ for all $r$.

Proof. The proof is just a careful re-examination of the argument of Heath-Brown and Testa [4]. This argument is based on a result of David [1] about successive minima for the quadratic form $Q$ corresponding to the canonical height on $\operatorname{Jac}(C)$. As in [4] (see (11)), 


$$
N(B) \ll \prod_{j \leq r} \max \left\{1,4 \frac{\sqrt{c \log B}}{M_{j}}\right\}
$$

where $c$ is an absolute constant and $M_{j}, j=1, \ldots, r$ are successive minima of $\sqrt{Q}$.

We now recall Corollary 1.6 from [1], which shows that if $D$ is the discriminant of $\operatorname{Jac}(C)$ then for all $l \leq r, M_{l} \gg(\log |D|)^{m_{l}}$, where $m_{l}=\frac{l^{2}-4 l-4}{8 l^{2}+8 l}$. Note that David's result refers to the successive minima for $Q$, while we have given the corresponding results for $\sqrt{Q}$.

In Lemma 4 we saw that $\|F\| \ll B^{30}$ if $N(B)>9$. There is, therefore, in that case an absolute constant $k$ such that

$$
\max \left\{1,4 \frac{\sqrt{c \log B}}{M_{j}}\right\} \leq k(\log B)^{1 / 2}(\log |D|)^{-m_{j}}
$$

for $j=1, \ldots, r$ since $\left|m_{j}\right|<1 / 2$ and $\log |D| \ll \log B$. Hence, if $N(B)>9$, then from (9) we obtain

$$
N(B) \ll k^{r}(\log B)^{r / 2}(\log |D|)^{-\left(m_{1}+\ldots+m_{r}\right)} .
$$

If $1 \leq r<16$, then $-\left(m_{1}+\ldots+m_{r}\right)>0$ and the assertion holds. If $r \geq 16$, let $D_{0}=\exp \left(k^{1 / m_{16}}\right)$. Then $k(\log |D|)^{-m_{j}} \leq 1$ for $j>16$ and $|D| \geq D_{0}$. Hence

$$
N(B) \ll(\log B)^{r / 2}(\log |D|)^{-\left(m_{1}+\ldots+m_{16}\right)} \ll(\log B)^{r / 2}
$$

as $-\left(m_{1}+\ldots+m_{16}\right)<0$. When $|D| \leq D_{0}$ the rank $r$ is bounded and we get the same assertion by (10).

So in any case, $N(B) \ll(\log B)^{r / 2}$, if $r \geq 16$. It should thereby be noted that Elkies (see [2]) has shown that there exist elliptic curves of rank $r \geq 28$.

\section{References}

[1] S. David, Points de petite hauteur sur les courbes elliptiques, J. Number Theory 64 (1997) $104-129$.

[2] N.D. Elkies, $\mathbb{Z}^{28}$ in $E(\mathbb{Q})$, May 2006, Number Theory Listserver.

[3] D.R. Heath-Brown, The density of rational points on curves and surfaces, Ann. of Math. (2) 155 (2002) 553-595.

[4] D.R. Heath-Brown, D. Testa, Counting rational points on cubic curves, Sci. China Math. 53 (9) (2010) 2259-2268.

[5] B. Mazur, Modular curves and the Eisenstein ideal, Inst. Hautes Études Sci. Publ. Math. 47 (1977) 33-186.

[6] P. Salberger, Counting rational points on projective varieties, preprint.

[7] G. Tenenbaum, Introduction to Analytic and Probabilistic Number Theory, Cambridge University Press, 1995. 